\title{
An Access Protocol for High-Speed Optical LANs*
}

\author{
Jonathan C. $\mathrm{Lu}$ and Leonard Kleinrock \\ Computer Science Department, University of California, Los Angeles
}

\section{Abstract}

In this paper a multiple access protocol is proposed for a system consisting of many high-speed bursty traffic stations via an optical passive star coupler. Each user has access over a range of wavelengths, thus resulting in a wavelength division multiplexed communication. A station has to reserve a wavelength first then transmits the data on that wavelength. The performance has been modeled and analyzed with the Equilibrium Point Analysis (EPA) technique. Numerical results show that low delay and high throughput (larger than the electronic speed of a single user) can be achieved. The analysis also shows that the best performance is obtained when the capacities of the reservation channels and the data channels are balanced.

\section{Introduction}

The explosive advance of fiber optics technology in the past decade offers a combination of wide bandwidth and low attenuation unmatched by any other transmission medium. It is conceivable that by using the low-loss passband of optical fibers $(1200-1600 \mathrm{~nm})$, we could construct multiple access networks carrying a total traffic of around 50 terabits per second [1]. An obstacle to realizing such high-speed transmission of optical signals is the bottleneck at the electronic interface. A fundamental limitation of single-channel high-speed networks such as FDDI [2] and DQDB [3] is that in these networks, the maximum throughput of the entire network is limited to the rate that can be supported by the electronics of one of the end user stations. Wavelength Division Multiple Access (WDMA) eliminates this bottleneck by operating on multiple channels at different wavelengths, with each channel running at a moderate

'This work wes supported by the Defense Advanced Research Projects Agency under Contract MDA 903-87-C0663, Parallel Systeme Laboratory.

Permiseion to copy without toe all or pert of this material is granted provided that the copies are not made or distributed for direct commercial advantage, the ACM copyright notice and the title of the publication and its date appear, and notice is given that copying is by permission of the Association for Computing Mrchinery. To copy otherwise, or to republish, requires a fee and/or specific pormission.

- 1992 ACM 089791-472-4/92/0002/0287 \$1.50 speed. However, the control of the WDMA system has proven to be a major obstacle and it is necessary to develop efficient medium access techniques for packet communications in this environment.

Today's electronically tunable semiconductor lasers and filters can tune from one wavelength to another in a few nanoseconds. However, the tuning range is limited. Therefore, each node can only operate on a small number of wavelengths [4]. One class of WDMA networks can be constructed by the use of fixed (wavelength) transmitters and fixed (wavelength) receivers. These networks employ multi-hop topologies in which a packet may be routed through several intermediate nodes before it is delivered to its destination. Examples of this type of networks can be found in $[5,6]$. The second class of WDMA networks assumes single-hop communications $[7,8]$ where multiple channels are created by employing tunable transmitters and/or receivers. In [7], a single tunable transmitter with limited tunability and multiple fixed receivers are provided to each station. A random time division multiple access (TDMA) protocol is used to determine which wavelength a station is allowed to transmit on. In [8], tunable filters (i.e., receivers) capable of rapid tuning over a large number of channels are assumed in order to support packet switching. Also, large packets have to be used since the slot (and thus the packet) size is proportional to the number of stations in the network.

The WDMA local area network can be realized using a bus or a star topology. A star topology is preferred [1] because the station-to-station link attenuation grows linearly with the number of stations. In a bus topology, the excess loss grows quadratically with the number of stations.

The system under consideration in this paper is a passive star network as shown in Figure 1. There are $(W+1)$ wavelengths available, $\lambda_{0}, \lambda_{1}, \ldots, \lambda_{W}$ to serve $N$ attached stations. The channel at wavelength $\lambda_{0}$ serves as the control channel for the exchange of the control traffic, while the other $W$ channels are for actual data traffic. Each station is equipped with two lasers: one fixed laser tuned at $\lambda_{0}$ and the other laser tunable to any of the wavelengths $\lambda_{1}, \ldots, \lambda_{w}$. The output of the two lasers is coupled into a $2 \times 1$ combiner, the output of which is connected to one of the inputs of the $N \times N$ star coupler. Signals transmitted at all of the $(W+$ 

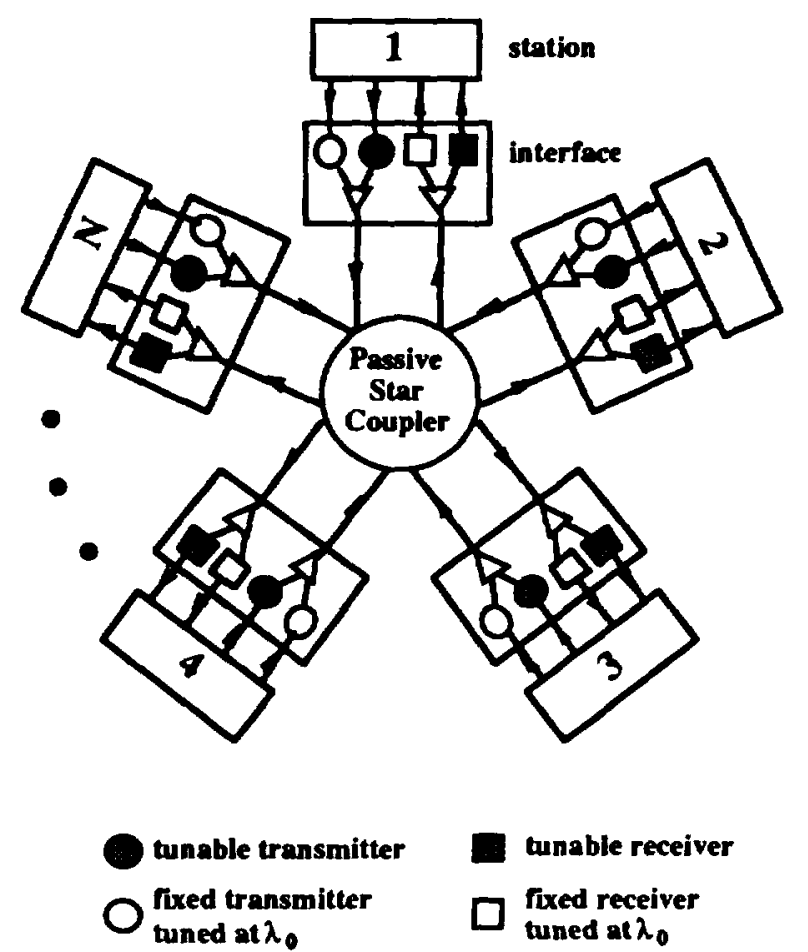

Figure 1: $N$ stations connected by a passive star coupler

1) wavelengths are combined at the star coupler and distributed to all of the stations. Each station also has two receivers: one fixed filter tuned at $\lambda_{0}$ and the other tunable to any of the wavelengths $\lambda_{1}, \ldots, \lambda_{W}$. At the receiver, the input optical signal is split into two parts by means of a $1 \times 2$ splitter. One part goes to the fixed optical filter which passes only the control wavelength $\lambda_{0}$, and the other output goes to the tunable filter which is tuned to pass the desired data wavelength.

In this paper, a multiaccess protocol based on reservation-ALOHA [9] is proposed and analyzed. In Section 2, we describe the details of the protocol. Section 3 presents the mathematical model and analysis. In Section 4, numerical results from both analysis and simulation are given. Section 5 concludes the paper.

\section{Description of Protocol}

\subsection{The Protocol}

We assume the existence of a common clock, obtained either by distributing a clock to all the stations or by means of some self-clocking mechanism inherent in the data. The problem of generating the global clock is addressed in [10]. Time is divided into slots. Packets are of fixed length, which is equal to one slot. The propagation delay from any station to the star coupler and then to any other station is assumed to be equal to $R$ slots. Slots on the data channels are called data slots

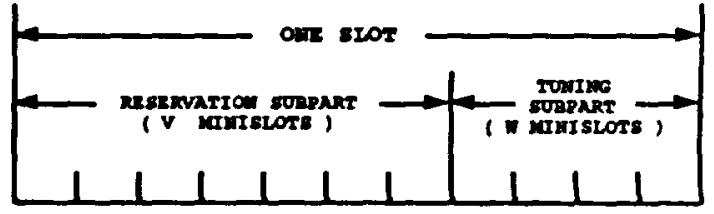

Figure 2: Structure of a control slot

and contain the actual data packets. Slots on the control channel are called control slots and carry only control information about the packets and the transmitters. Each control slot consists of a reservation subpart and a tuning subpart. The reservation subpart is divided into $V$ minislots to be used on a contention basis with the slotted ALOHA protocol, and the tuning subpart is divided into $W$ minislots to convey the wavelength tuning information. The structure of a control slot is shown in Figure 2.

A station generating a packet will randomly select one of the $V$ reservation minislots in the next control slot and transmit a reservation minipacket on the control channel. $R$ slots later the station will hear the result of its reservation. If it is successful, it is received by all the stations because of the broadcast nature of the control channel. All successful reservations join a common distributed queue of stations waiting to transmit. If there is a collision, the station will transmit another reservation minipacket in the next slot with probability $p$, and with probability $(1-p)$ it will defer the decision by one slot and transmit the reservation in this next slot with probability $p$, etc.

In the tuning subpart of each control slot, each of the first $W$ stations in the distributed queue will transmit a tuning minipacket in an assigned tuning minislot; the minipacket contains the destination address for the data packet to be transmitted in the next slot. In particular, the first station in the queue transmits its minipacket in the first tuning minislot, the second station in the queue transmits in the second minislot, $\ldots$, and the $W$ th station transmits in the $W$ th minislot. The position of the tuning minislot uniquely determines the wavelength to use. At the beginning of the next slot, those $W$ stations tune their tunable transmitters to their assigned wavelengths, with the ith station in the queue using wavelength $\lambda_{i}$ to transmit its data packet. When the destination sees its address announced in a tuning minislot on the control channel, it tunes its tunable receiver to the corresponding wavelength and receives the data packet at the beginning of the next slot. If two or more packets are addressed to the same destination in a slct, we arbitrarily select the one transmitted on the lowest wavelength number to win the competition. The losing stations must start over with the reservation procedure again.

Thus, a station desiring to send a packet must first 
compete on the ALOHA reservation subchannel to gain access to a minislot on the tuning subchannel. The station then informs its intended receiver to listen (i.e., tune) to a particular wavelength on which the data packet will be transmitted. If a given receiver is informed by more than one station, only one station will be selected, and the others must repeat the entire procedure.

Note that there is a minimum of one round trip delay before a data packet can be transmitted even at light load. A modification of the protocol can be made as follows to improve this: If the current length of the distributed queue, $C$, is smaller than $W$ and a station decides to transmit a reservation, then the station, instead of transmitting a reservation minipacket, will randomly pick one among those $(W-C)$ free tuning minislots to send out a tuning minipacket, and transmit the data packet on that chosen wavelength at the beginning of next slot. If more than one station chooses the same tuning minislot, a collision occurs and all the stations involved must repeat this procedure provided $C$ is still smaller than $W$. Otherwise they must send out reservations first and follow the basic protocol. Thus the data packets need not suffer from the round trip delay due to making reservations and the performance at light load is much improved. This modification is not included in the analytic treatment in the following sections.

\section{Performance Analysis}

\subsection{Model Assumptions}

We assume that there are $(W+1)$ wavelenghts available and the number of stations in the network is $N$. Each station has a single buffer which is equal to the size of a packet. A new packet arrives at a station with an empty buffer with probability $\sigma$ at the end of a slot. A packet generated by a station is addressed to any of the other $(N-1)$ stations with equal probability. A source station with a full buffer (i.e., one packet) will not discard its packet until its successful reception at its destination is recognized.

\subsection{An Approximate Model}

An approximate model of the system is shown in Figure 3. In this model, each station can be in one of the following $(3 R+3)$ modes at any instant: $T H, R T, Q, P Q_{m}, P R_{m}$, and $P S_{m}(1 \leq m \leq R)$. Each station can move from one mode to another mode only at the beginning of each slot.

Stations in each mode act as follows. Stations in the $T H$ (thinking) mode generate a packet with probability $\sigma$ at the end of a slot. Stations in the $Q$ (queued) mode are currently in the distributed queue. A station that had suffered a collision of its reservation packet and has realized it is said to be in the $R T$ (retransmission) mode and will retransmit the reservation with probability $p$ in the next slot. The $P Q_{m}, P R_{m}$ and $P S_{m}$ modes are unit delay elements, which express the influence of the channel propagation delay. Stations in the $P Q_{m}$ mode will move into the $P Q_{m-1}$ mode at the next slot with probability 1. Thus, as can be seen in Figure 3, stations in the $P Q_{m}$ mode will enter the $Q$ mode after $m$ slots. The transistions of stations in the $P R_{m}$ and $P S_{m}$ modes are defined similarly.

We now define a state vector of the system. Let $n_{R r}$ be a random variable representing the number of stations in the $R T$ mode, $n_{Q}$ that in the $Q$ mode, $i_{m}$ that in the $P R_{m}$ mode, $j_{m}$ that in the $P Q_{m}$ mode, and $k_{m}$ that in the $P S_{m}$ mode, $m=1, \ldots, R$. In the model we will further make a nonpersistence assumption : a station, upon entering the $R T$ mode, will randomly reselect a destination for its packet (It is not the case in the real system, but later we will see that the model still predicts the performance very well under this assumption). Define the vector $\mathbf{n} \triangleq$ $\left(n_{R r}, n_{Q}, i_{1}, \cdots, i_{R}, j_{1}, \cdots, j_{R}, k_{1}, \cdots, k_{R}\right)$ as the state vector of the system. Then we can see that the vector $\mathbf{n}$ forms a discrete-time Markov chain with a finite state space.

Unfortunately, since the state space is so large, it is difficult for us to solve this Markov chain. Therefore, we utilize the technique of equilibrium point analysis (EPA) [15] to analyze this chain.

\subsection{The Modified Model}

For simplicity of analysis, we first consider a modification of the model in Figure 3 as suggested in [15], which combines the two inputs (from the $T H$ mode and the $R T$ mode) of the slotted ALOHA reservation channel into one. Since we have assumed bursty users, we shall confine ourselves to the case $\sigma \leq p$. The modified model is shown in Figure 4, where the TH mode in Figure 3 has been decomposed into two modes, $I$ and $T$, and the $R T$ mode in Figure 3 has become part of the $T$ mode. A station that has just moved out from the $P S_{1}$ mode moves into the $I$ and the $T$ modes with probabilities $(1-\sigma / p)$ and $\sigma / p$, respectively. A station in the $I$ mode will move into the $T$ mode at the next slot with probability $\sigma$, and a station in the $T$ mode transmits a reservation minipacket (i.e., moves out from the $T$ mode) with probability $p$. The model in Figure 4 is equivalent to that in Figure 3 from the viewpoint of the stochastic behavior to be explained below; consequently, we can derive any characteristic of the model in Figure 3 by using the model in Figure 4.

The equivalence of the two models can be interpreted as follows: Let $X_{1}, Y_{1}$, and $Y_{2}$ be random variables rep- 


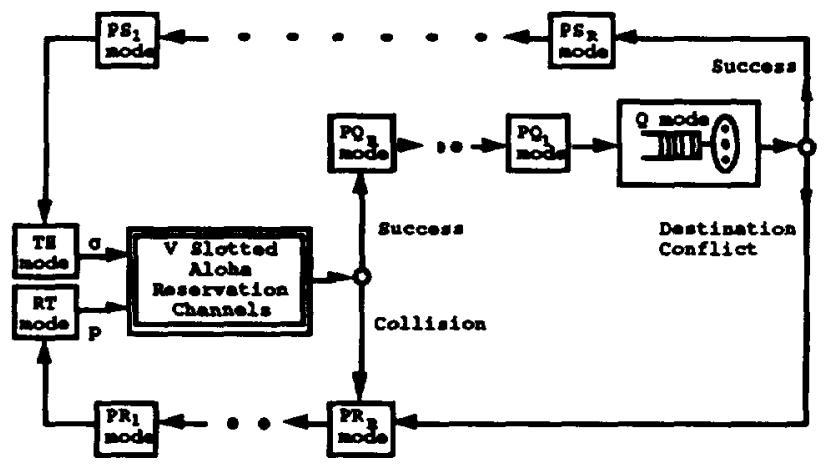

Figure 3: An approximate model of the system

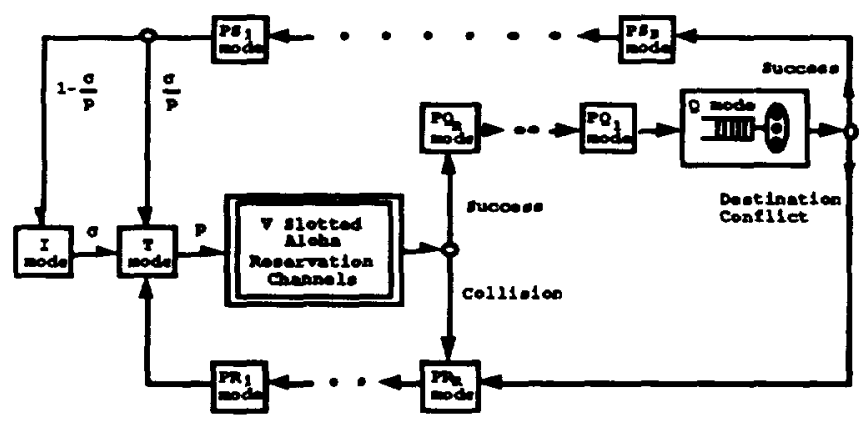

Figure 4: A modified model of Figure 5 in the case of $\sigma \leq p$

resenting the time (number of slots) during which a station is in the $T H, R T$, and $T$ modes, respectively. Also let $X_{2}$ be a random variable representing the time from the moment a user enters either the $I$ mode or the $T$ mode from the $P S_{1}$ mode until the instant the station moves out of the $T$ mode. (Note that $X_{2}$ corresponds to $X_{1}$.) It can be shown [15] that $X_{2}$ and $Y_{2}$ have the same pmf's as $X_{1}$ and $Y_{1}$, respectively.

In the modified model in Figure 4, we now let $n_{T}$ be a random variable representing the number of stations in the $T$ mode. Then, it is apparent that the modified state vector $\mathbf{n}=\left(n_{T}, n_{Q}, i_{1}, \cdots, i_{R}, j_{1}, \cdots, j_{R}, k_{1}, \cdots, k_{R}\right)$ is also a Markov chain under the nonpersistence assumption.

\subsection{The Equilibrium Point Equation}

An equilibrium point is a point in state space such that at that point the expected increase in the number of stations in each mode per unit time is zero [16]. In the EPA method, we assume that the system is always at an equilibrium point. Applying the above condition to all the modes, we get a set of equations called equilibrium point equations, whose solution gives one or more equilibrium points.

Let $\overline{\mathbf{n}}=\left(\bar{n}_{r}, \bar{n}_{\theta}, \bar{i}_{1}, \cdots, \bar{i}_{R}, \bar{j}_{1}, \cdots, \bar{j}_{R}, \bar{k}_{1}, \cdots, \bar{k}_{R}\right)$ be an equilibrium point. Let $\delta_{I}(\overline{\mathbf{n}})$ denote the conditional expectation of the increase in the number of stations in the $I$ mode in a slot, given that the system is at $\overline{\mathbf{n}}$. Setting $\delta_{I}(\overline{\mathbf{n}})=0$, we then have

$$
\begin{aligned}
\delta_{I}(\overline{\mathbf{n}}) & =\bar{k}_{1}\left(1-\frac{\sigma}{p}\right) \\
& -\left[N-\bar{n}_{T}-\bar{n}_{Q}-\sum_{m=1}^{R}\left(\bar{i}_{m}+\bar{j}_{m}+\bar{k}_{m}\right)\right] \sigma=0
\end{aligned}
$$

Next, let $X(\overline{\mathbf{n}})$ denote the conditional expectation of the number of stations that move out from the $Q$ mode in a slot, given that the system is in state $\overline{\mathbf{n}}$. Evaluating mode $Q$, we get

$$
\delta_{Q}(\overline{\mathbf{n}})=X(\overline{\mathbf{n}})-\bar{j}_{1}=0
$$

Next we define two other terms. Let $f_{T}(\overline{\mathbf{n}})$ denote the conditional expectation of the number of stations that successfully transmit reservation minipackets (thus move from mode $T$ to mode $P Q_{R}$ ) in a slot, given that the system is in state $\overline{\mathbf{n}}$. Let $g_{Q}(i)$ denote the average number of stations that successfully transmit a data packet (therefore moving from mode $Q$ to mode $P S_{R}$ ) in a slot, given that $i$ stations transmit (i.e. move cut of mode $Q$ ). It can be derived [12] that

$$
f_{T}(\overline{\mathbf{n}})=\bar{n}_{T} p\left(1-\frac{p}{V}\right)^{\bar{n}_{T}-1}
$$

and

$$
g_{Q}(i)=N\left[1-\left(1-\frac{1}{N-1}\right)^{i-1}\left(\frac{N^{2}-2 N+i}{N(N-1)}\right)\right]
$$

Evaluating the conditional expectation of increase for the $P R_{m}, P Q_{m}$, and $P S_{m}(1 \leq m \leq R)$ modes, we obtain the corresponding equations as follows:

$$
\begin{gathered}
\bar{i}_{1}=\bar{i}_{2}=\cdots=\bar{i}_{R}=\bar{n}_{T} p-f_{T}(\overline{\mathbf{n}})+X(\overline{\mathbf{n}})-g_{Q}(X(\overline{\mathbf{n}})) \\
f_{T}(\overline{\mathbf{n}})=\bar{j}_{R}=\cdots=\bar{j}_{1} \\
\bar{k}_{1}=\cdots=\bar{k}_{R}=g_{Q}(X(\overline{\mathbf{n}}))
\end{gathered}
$$

We did not write down the equation for the $T$ mode above since it is linearly dependent on the others.

We model the queueing system in mode $Q$ as a $W$ server system with a binomial input with mean $f_{T}(\overline{\mathbf{n}})$ and a fixed one slot service time for each customer. Define

$$
\rho=\frac{f_{\tau}(\overline{\mathbf{n}})}{W}
$$

which is the utilization of the queueing system. Define $A_{t}$ as the number of customers that arrive between time $t-1$ and $t$. Let $A \triangleq \lim _{t \rightarrow \infty} A_{t}$. The probability mass function (pmf) and $z$-transform of $A$ are 


$$
\begin{gathered}
a_{j} \triangleq \operatorname{Prob}[A=j]=\left(\begin{array}{c}
V \\
j
\end{array}\right)\left(\frac{\rho W}{V}\right)^{j}\left(1-\frac{\rho W}{V}\right)^{V-j} \\
A(z) \triangleq \sum_{j=0}^{V} a_{j} z^{j}=\left(1-\frac{\rho W}{V}+\frac{\rho z W}{V}\right)^{V}
\end{gathered}
$$

Define $C_{t}$ as the number of customers in the system at time $t$. Let $C \triangleq \lim _{t \rightarrow \infty} C_{t}$. We first have

$$
C_{t+1}=\max \left(0, C_{t}+A_{t+1}-W\right)
$$

Assume that steady state exists. Solving this using the technique in [13], we get

$$
C(z)=E\left[z^{C}\right]=\frac{\left(1-z_{1}\right)\left(1-z_{2}\right) \cdots\left(1-z_{(V-W)}\right)}{\left(z-z_{1}\right)\left(z-z_{2}\right) \cdots\left(z-z_{(V-W)}\right)}
$$

where $z_{i}, i=1, \ldots,(V-W)$, are the roots of $A(z)-$ $z^{W}=0$ outside the unit circle $|z|=1$. The average number of customers in the system should be approximately equal to $\bar{n}_{\boldsymbol{Q}}$, the average number of stations in mode $Q$. Therefore, we have

$$
\bar{n}_{Q}=E[C]=-\sum_{i=1}^{V-W} \frac{1}{1-z_{i}}
$$

Also, since $X(\overline{\mathbf{n}})=f_{T}(\overline{\mathbf{n}})=\rho W$, from equations (1)-(5) we have

$$
\begin{gathered}
\bar{n}_{r} p\left(1-\frac{p}{V}\right)^{\bar{n}_{r-1}}-\rho W=0 \\
\frac{g_{Q}(\rho W)}{\sigma}\left(1-\frac{\sigma}{p}\right)=N-(1+p R) \bar{n}_{T}-\bar{n}_{Q}-\rho R W
\end{gathered}
$$

It is apparent that the equations (6) (7) and (8) can be solved for $\rho$. The system is said to be stable if only one solution exists. Otherwise, if there is more than one solution, the system is said to be unstable [16].

\subsection{Throughput and Delay}

Let us now define the throughput $S(\mathbf{n})$ to be the conditional expectation of the number of correctly transmitted data packets in a slot, given that the system is in state $\mathbf{n}$. Then, it is clear that the throughput at an equilibrium point is expressed as

$$
S(\overline{\mathbf{n}})=g_{\bullet}(X(\overline{\mathbf{n}}))=g_{\bullet}(\rho W)
$$

The average packet delay, which is the average time, in number of slots, from the moment the packet is generated until the instant the packet is correctly received by the destination, can be calculated from Little's result [13] to give

$$
E[D]=\frac{N}{S(\overline{\mathbf{n}})}-\frac{1}{\sigma}
$$

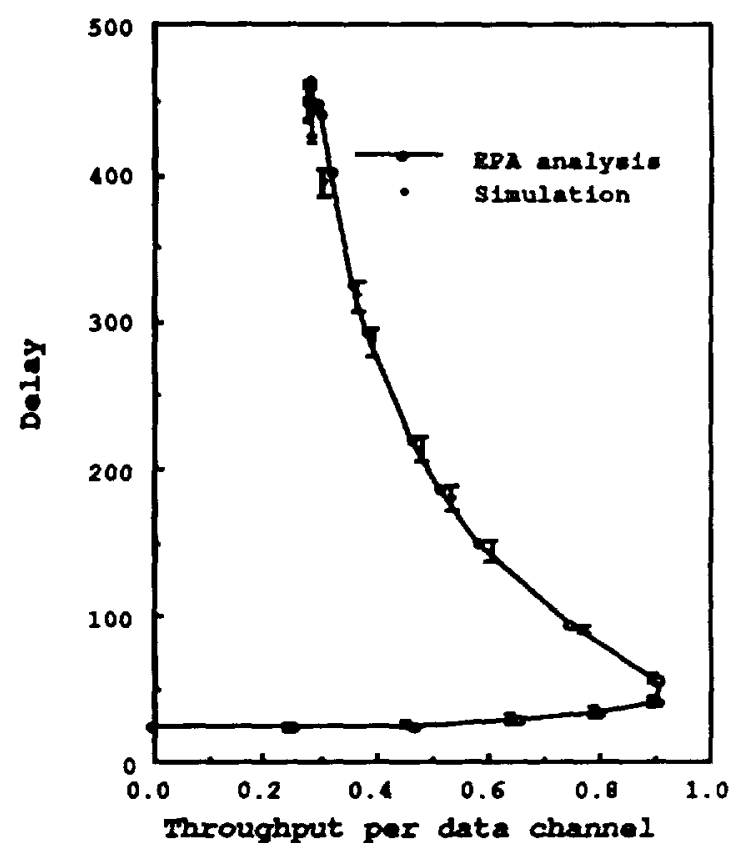

Figure 5: Throughput per data channel versus delay. $N=500, W=4, V=10, R=10, p=0.2$.

\section{Numerical Results}

In this section we use both the analytical model and simulation to investigate a specific system consisting of 500 stations interconnected through 5 wavelengths $(W=$ 4 in this case). The propagation delay $R$ is equal to 10 slots. In all figures, we note the excellent agreement between the analysis and simulation.

Figure 5 shows the throughput per data wavelength versus delay curve for $V=10, p=0.2$. and $\sigma$ increasing from 0 to 0.2 (the 95 per cent confidence intervals of the simulation results are also shown in the figure). Note that in the lower part of the curve the delay increases very slowly with the throughput; thus a high throughput per data channel and low delay can be achieved.

Figures 6 and 7 show the effect of varying $V$ and $W$ while keeping their sum constant by fixing the slot size. We can see that for different cases the maximum throughput always occurs at the point where $V \approx e W$. This is not surprising since the capacity of a slotted ALOHA channel is $1 / e$. By making $V \approx e W$ the capacities of the $V$ ALOHA channels and the $W$ data channels are balanced. When $V \ll e W$, there is not enough throughput coming out of the ALOHA reservation channels to keep the $W$ data channels busy. When $V \gg e W$, most stations are waiting (in the $Q$ mode) for a wavelength on which to transmit a data packet. When $V \approx e W$, the throughput is roughly equal to the load offered by the stations.

Let $D_{T}$ and $D_{Q}$ denote the average time a station 
spends in modes $T$ and $Q$, respectively, in a cycle. By Little's result, the throughput is equal to $N /\left(\frac{1}{\sigma}+D_{T}+\right.$ $R+D_{Q}+R$ ). Setting both $D_{T}$ and $D_{Q}$ to 1 , which is the minimum possible value, we obtain an upper bound for the throughput,

$$
S_{U B}=\frac{N}{\frac{1}{\sigma}+2(R+1)}
$$

and a lower bound for the delay,

$$
D_{M I N}=\frac{N}{S_{U B}}-\frac{1}{\sigma}=2(R+1)
$$

which are the "flat" regions in Figures 6 and 7 , respectively. Note that when $S_{U B}>\min (V / e, W)$ (the case of $V+W=14$ ), the flat region disappears since the throughput is not limited by the user-generated load.

The effect of varying the propagation delay, $R$, is shown in Figures 8 and 9. Although the physical distance of the network is usually fixed, $R$ can be varied by varying the packet (and thus the slot) size. We see that when $R$ is small, too much traffic is offered to the $V$ ALOHA reservation channels and the throughput is small. As we increase $R$, the throughput increases too. It reaches the maximum at $R=7$, then falls off because there is not enough traffic in each slot when $R$ becomes large. The tail of the throughput curve is bounded by the upper bound $S_{U_{B}}$. The reason the increase is so sharp around $R=6$ and 7 is that the system changes from an overloaded system (see Figure 7 (d) in [16]) to a bistable one and then to a stable one as $R$ increases from 5 to 7. This phenomenon is observed in both the EPA analysis and the simulation. (For the case $R=$ 6 , the average value of the two solutions obtained from EPA is plotted.)

Figure 10 shows the influence of the destination conficts. Suppose $i$ data packets are transmitted in a slot. The number of packets successfully received by their destinations is $g_{0}(i)$. We plot the fraction of success, $g_{Q}(i) / i$, versus $i$ assuming $N=500$. Near-term technology limits $W$, the number of transmitter/receivertunable wavelengths available, to be fewer than about twenty; thus we see that destination conflicts are not a serious concern.

\section{Conclusions}

In this paper a wavelength division multiple access protocol (with $W$ wavelength channels) was proposed to provide a high-capacity optical fiber local area network to a large population of $N$ users. We assumed $N \geq W$. The users' traffic was assumed to be bursty as in the case of computer communications. The performance of the protocol was completely modeled and analyzed. Numerical results show that an aggregate throughput substantially larger than the electronic speed of a single

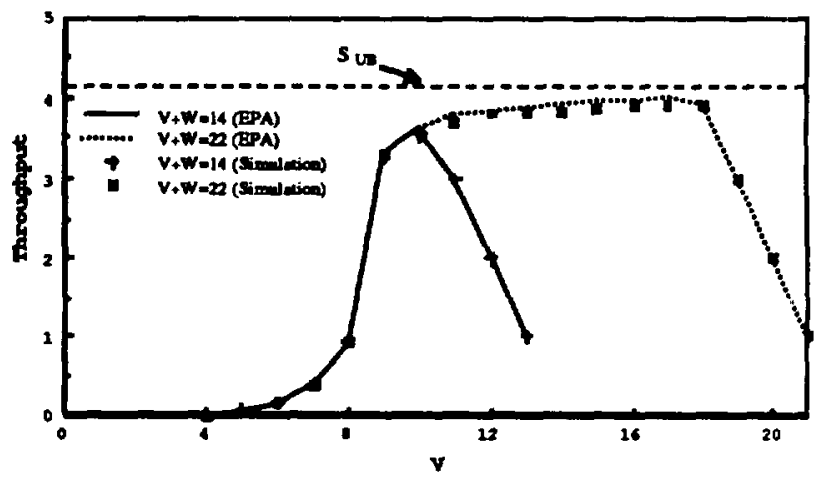

Figure 6: Throughput versus $V$ for fixed slot sizes $(V+$ $W$ constant). $N=500, R=10, \sigma=0.01, p=0.2$.

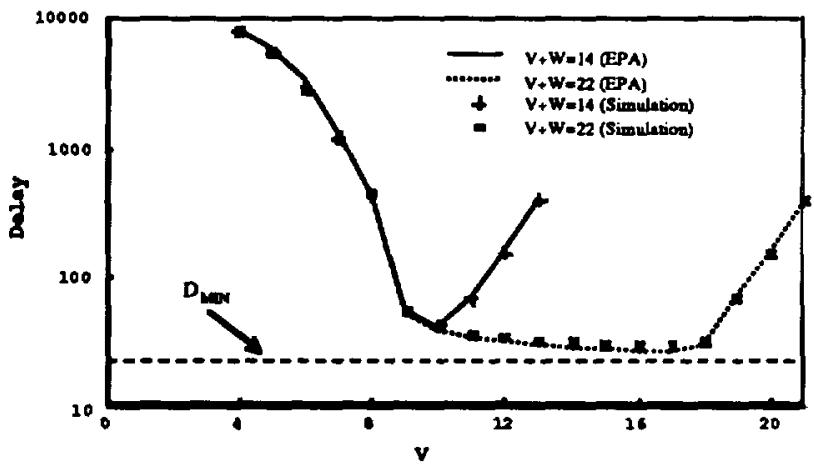

Figure 7: Delay versus $V$ for fixed slot sizes $(V+W$ constant). $N=500, R=10, \sigma=0.01, p=0.2$.

station can be supported. The effects of various system parameters and their optimal selection were also investigated. By comparison with simulation, our analytical approximations were shown to be excellent.

\section{References}

[1] P. S. Henry, "High-capacity lightwave local area networks," IEEE Commun. Mag., vol. 27, pp. 20 26, Oct. 1989.

[2] F. E. Ross, "FDDI - A tutorial," IEEE Commur.. Mag., vol. 24, May 1986.

[3] R. M. Newman, Z. L. Budrikis, and J. L. Hullet, "The QPSX MAN", IEEE Commun. Mag., vol. 26, pp. 20 - 28, Apr. 1988.

[4] C. A. Brackett, "Dense wavelength division multiplexing networks: Principles and applications," 


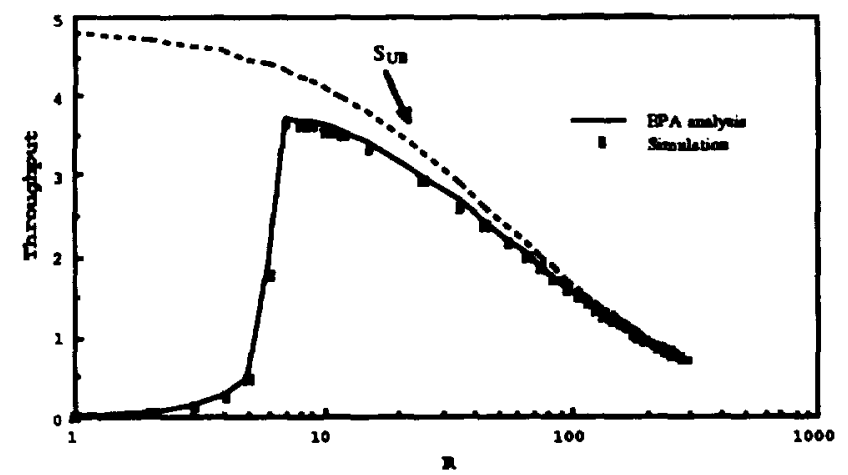

Figure 8: Throughput versus propagation delay. $N=$ $500, W=4, V=10, \sigma=0.01, p=0.2$.

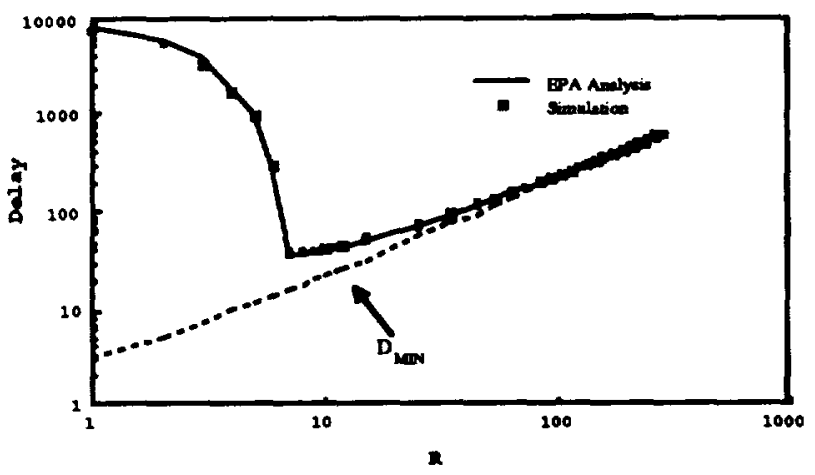

Figure 9: Delay versus propagation delay. $N=500$, $W=4, V=10, \sigma=0.01, p=0.2$.

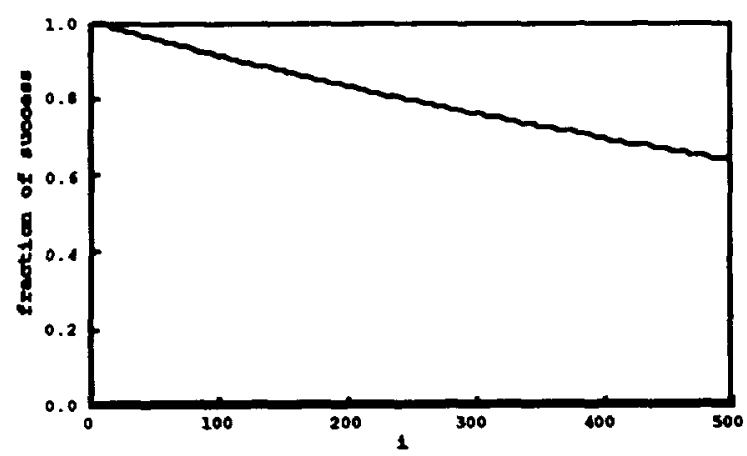

Figure 10: The fraction of success versus the number of data packets transmitted. $N=500$.
IEEE J. Select. Areas. Commun., vol. 8, no. 6, pp. 948 - 964, Aug. 1990.

[5] M. G. Hluchyj and M. J. Karol, "ShuffleNet : An application of generalized perfect shuffles to multihop lightwave networks," pp. 4B.4.1 - 4B.4.12, Infocom '88, 1988.

[6] 1. Chlarntac and A. Ganz, "Toward alternative high-speed network concepts: The SWIFT architecture," IEEE Thans. Commun., vol. COM-38, pp. 431 - 439, Apr. 1990.

[7] A. Ganz and Z. Koren, "WDM passive star - Protocols and performance analysis," pp. 9A.2.1 9A.2.10, Infocom '91, 1991.

[8] M. -S. Chen, N. R. Dono, and R, Ramaswami, "A media-access protocol for packet-switched wavelength division multiaccess metropolitan area networks," IEEE J. Select. Areas. Commun., vol. 8, no. 6, pp. 1048 - 1057, Aug. 1990.

[9] L. G. Roberts, "Dynamic allocation of satellite capacity through packet reservation," in AFIPS Conf. Proc., vol. 42, pp. $711-716,1973$.

[10] Y. Ofek and M. Sidi, "Design and analysis of a hybrid access control to an optical star using WDM," pp. 2A.3.1 - 2A.3.12, Infocom '91, 1991.

[11] L. Kleinrock, Queueing Systems, Vol. II: Computer Applications, John Wiley and Sons, New York, 1976.

[12] J. C. Lu, "Design and analysis of Wavelength Division Multiple Access Lightwave Networks," Ph.D. dissertation, Comput. Sci. Dept., Univ. California, Los Angeles, 1992.

[13] L. Kleinrock, Queueing Systems, Vol. I: Theory, John Wiley and Sons, New York, 1975.

[14] S. S. Lam, "Packet switching in a multi-access broadcast channel with applications to satellite communication in a computer network," Computer Science Department, School of Engineering and Applied Science, Engineering Report UCLA-ENG7429, Mar. 1974.

[15] S. Tasaka and $Y$. Ishibashi, "A reservation protocol for satellite packet communication - A performance analysis and stability considerations," IEEE Trans. Commun., vol. COM-32, pp. 920 - 927, Aug. 1984.

[16] L. Kleinrock and S. S. Lam, "Packet switching in a multiaccess broadcast channel: Performance evaluation," IEEE Trans. Commun., vol. COM-23, pp. $410-423$, Apr. 1975. 
